\title{
Ativação Muscular e Determinação do Limiar de Fadiga Eletromiográfico Durante Teste de Carga Incremental em Cicloergômetro de Braço: um estudo piloto
}

\author{
Felipe Bergh \\ Ivan Luís Andrade \\ Pierre Ferreira \\ Orientador Eduardo Fontes \\ Tipo de Análise do Manuscrito Triple Blind Peer Review \\ Recebido em Nov/2011 Aprovado em Dez/2011
}

\section{Resumo}

O objetivo do presente estudo foi verificar a ativação de quatro músculos do membro superior (Bíceps Braquial [BB], Tríceps Braquial [TB], Deltóide Anterior [DA] e Deltóide Posterior [DP]) e determinar o Limiar de Fadiga Eletromiográfico (LFEMG) durante um teste incremental em ergômetro de braço. Quatro voluntários do sexo masculino (idade: 25,25 \pm 4,7; estatura: $173 \pm 4 \mathrm{~cm}$; massa corporal: 71,5 $\pm 6,3 \mathrm{~kg}$ ) realizaram um teste incremental com rotação aproximada de $80 \mathrm{rpm}$ até atingir a potência máxima do ergômetro (incrementos de carga de 3-4 W a cada 4 min, começando com $19 \mathrm{~W}$ e terminando com 48 W). 0 sinal eletromiográfico (EMG) foi coletado durante os testes (Eletromiógraf o Myosystem 2000, Noraxon, EUA) e posteriormente tratado com uso do programa MATLAB 7.7.0 (retificação e cálculo do RMS). O LFEMG foi detectado a partir de inspeção visual. Foram também registrados os valores de freqüência cardíaca (FC) no término de cada estágio. Houve grande variação da ativação muscular entre os músculos analisados durante o teste incremental. DA, DP e TB tiveram um comportamento de queda do sinal EMG, já o BB apresentou aumento no decorrer do teste. O LFEMG foi encontrado apenas no músculo BB (Potência: 44 W, FC: 123 bpm) de um sujeito, representando aproximadamente 90\% da potência máxima do ergômetro. Nossos resultados sugerem que o aumento da carga imposta sobre o movimento cíclico bilateral exigido pelo cicloergômetro de braço ocasion ou uma ação compensatória evidenciada pelo aumento da ativação do BB e diminuição do TB. Já a queda de ativação dos músculos estabilizadores do ombro (DA e DP) pode estar associada à indícios de fadiga periférica e/ou aproveitamento do torque gerado pelo $\mathrm{BB}$. 0 presente estudo aponta ainda queo BB é o músculo com maior probabilidade de apresentar o LFEMG em um teste incremental.

Palavras-chave: Eletromiografia, Ativação Muscular, Fadiga, Ergômetro de braço

\section{Muscle Activation and Determination of Electromyographic Fatigue Threshold During Incremental Load Test in Arm Ergometer: a pilot study}

\section{Abstract}

The aim of this study was to assess the activation of four muscles of the upper limb (Biceps Brachii [BB], Triceps Brachii [TB], Anterior Deltoid [AD] and Posterior Deltoid [PD]) and 
determine the electromyographic fatigue threshold (EMGth) during an incremental test on an arm ergometer. Four male volunteers (age: $25.25 \pm 4.7$, height: $173 \pm 4 \mathrm{~cm}$, body mass: $71.5 \pm$ $6.3 \mathrm{~kg}$ ) performed an incremental test with an approximate cadence of $80 \mathrm{rpm}$ until they reach the maximum power output of the ergometer (load increments of 3-4 W every 4 min, starting with $19 \mathrm{~W}$ and ending at $48 \mathrm{~W}$ ). The electromyographic signal (EMG) was collected during the tests (Myosystem 2000 Noraxon, USA) and subsequently treated using the program MATLAB 7.7.0 (correction and calculation of RMS). The EMGth was detected from visual inspection. Values of heart rate (HR) were also recorded at the end of each stage. There was great variation in muscle activation between the muscles examined during the incremental test. AD, PD and TB had a decreasing behavior of the EMG signal, however, $\mathrm{BB}$ showed an increase during the test. The EMGth was found only in the BB muscle (power: 44 W, HR: $123 \mathrm{bpm}$ ) of one subject, representing approximately $90 \%$ of the maximum power of the ergometer. Our results suggest that increasing the workload on the cyclical movement required by the bilateral arm ergometer caused a compensatory action evidenced by increased activation of BB and decreased of TB. Already the decay of activation of stabilizing muscles of the shoulder (DA and DP) can be associated with signs of peripheral fatigue and / or use of the torque generated by the BB.. This study also shows that BB is the muscle most likely to reach the EMGth in an incremental test.

Keywords: Electromyography. Muscle Activation. Fatigue. Arm Ergometer

\begin{abstract}
Activación Muscular y Determinación de Umbral de la Fatiga Electromiográfica Durante Test de Carga Incrementales em Ergómetro De Brazos: un estudio piloto
\end{abstract}

\title{
Resumen
}

El objetivo de este estudio fue evaluar la activación de los cuatro músculos del miembro superior (bíceps braquial [BB], Triceps braquial [TB], anterior del deltoides [AD] y posterior Deltoides [PD]) y determinar el umbral de la fatiga electromiográfica (UFemg) durante uma prueba incremental en un ergómetro de brazos. Cuatro voluntarios varones (edad: 25,25 \pm 4,7 , altura: $173 \pm 4 \mathrm{~cm}$, masa corporal: $71,5 \pm 6,3 \mathrm{~kg}$ ) realizaron un test incremental con uma velocidad aproximada de $80 \mathrm{rpm}$ hasta que alcanza la potencia máxima del ergómetro (los incrementos de carga de 3-4 W cada 4 minutos, a partir de $19 \mathrm{~W}$ y finaliza a $48 \mathrm{~W}$ ). El señal electromiográfico (EMG) fue recogida durante las pruebas (Myosystem Noraxon 2000, EE.UU.) y posteriormente tratado mediante el programa MATLAB 7.7.0 (corrección y el cálculo de RMS). El UFemg se detectó tras una inspección visual. También se registraron valores de frecuencia cardíaca (FC) al final de cada etapa. Hubo una gran variación en la activación muscular entre los músculos examinados durante el test incremental. AD, PD y TB tuvo un comportamiento decreciente de la señal de EMG, ya que el BB presentó un aumento en el transcurso de la prueba. El UFemg se encontró sólo en el músculo BB (potencia: $44 \mathrm{~W}$, FC: $123 \mathrm{bpm}$ ) de un sujeto, lo que representa aproximadamente el $90 \%$ de la potencia máxima del ergómetro. Nuestros resultados sugieren que el aumento de la carga sobre el movimiento cíclico bilateral requerido por el ergómetro de brazos causó una acción compensatória evidenciado por una mayor activación de BB y disminución de TB. Ya la caída de la activación de los músculos estabilizadores del hombro (DA y DP) puede estar asociada com signos de fatiga periférica, y / o uso del torque generado por BB. Este estudio también muestra que el BB es el músculo con mayor probabilidad de presentar el UFemg en un test incremental.

Palabras-Clave: Electromiografía. Activación Muscular. Fatiga. Ergómetro de Brazos.

\section{Activation des muscles et détermination du seuil de fatigue électromyographique Pendant bras de test de charge progressive du cycle ergomètre: une étude pilote}

\section{Résumé}

Le but de cette étude était d'étudier l'activation de quatre muscles des membres supérieurs (biceps [BB], triceps [TB], deltoïde précédente [DA] et deltoïde postérieur [SD]) et de déterminer le seuil de fatigue électromyographique (LFEMG) lors d'un test supplémentaire dans ergomètre pour les bras. Quatre volontaires de sexe masculin (âge: $25,25 \pm 4,7$, hauteur: $173 \pm 4 \mathrm{~cm}$, la masse corporelle: $71,5 \pm 6,3 \mathrm{~kg}$ ) a effectué un test incrémental avec une vitesse 
approximative de 80 tours par minute pour atteindre la puissance maximale l'ergomètre (incréments de charge de $3-4 \mathrm{~W}$ toutes les 4 min à partir de $19 \mathrm{~W}$ et se terminant avec $48 \mathrm{~W}$ ). Le électromyographie (EMG) ont été recueillies pendant les essais (électromyogramme Myosystem 2000, Noraxon, USA) et ensuite traité avec l'utilisation de MATLAB 7.7.0 (de rectification et de calcul de RMS). Le LFEMG a été détectée à partir de l'inspection visuelle. Les valeurs ont également été enregistrées fréquence cardiaque $(\mathrm{FC})$ à la fin de chaque étape. Il y avait une grande variation dans l'activation des muscles entre les muscles analysés lors de l'essai supplémentaire. $A D, P D$ et de la tuberculose a fait une chute le comportement du signal EMG, puisque BB a augmenté pendant le test. Le LFEMG a été trouvé seulement dans le muscle BB (sortie: $44 \mathrm{~W}$, HR: $123 \mathrm{bpm}$ ) d'un sujet, ce qui représente environ 90\% de la puissance maximale de l'ergomètre. Nos résultats suggèrent que l'augmentation de la charge sur le mouvement cyclique requis par ergomètre pour les bras bilatérale causé une action compensatoire en évidence par l'activation accrue de la BB et la tuberculose a diminué. Depuis la chute de l'activation des muscles de l'épaule de stabilisation (AD et PD) peut être associée à des signes de fatigue périphérique et / ou l'utilisation du couple généré par le BB. Cette étude montre également queo BB muscle est plus susceptible d'avoir le LFEMG dans un test supplémentaire.

Mots-clés: électromyographie, activation musculaire, fatigue, ergomètre pour les bras

$*$

\section{Introdução}

A eletromiografia de superfície (EMG) tem sido bastante utilizada para verificar o recrutamento de músculos durante uma atividade física e a ocorrência de fadiga neuromuscular (SILVA \& GONÇALVES, 2003). 0 método é bastante viável e prático, pois além de simples de ser manuseado pelo pesquisador, é não-invasivo e não apresenta desconforto para o sujeito a ser analisado. A EMG vem sendo utilizada na área clínica (BARBOSA \& GONÇALVES, 2005), bem como para estudos de análises biomecânicas (GABRIEL, BASFORD \& AN, 2001) e fisiológicas (HUG et al., 2003).

Durante um esforço incremental, o recrutamento adicional de fibras é evidenciado (OLIVEIRA et al., 2005) e vem sendo sugerido que esse comportamento ocorre a fim de compensar a perda de força pelas fibras fadigadas (GONÇALVES, 2006). Esse padrão de

recrutamento pode ser verificado pelo aumento da atividade de EMG. De acordo com o incremento da intensidade do exercício, se estabelece uma relação linear entre a Raiz do Quadrado Médio (Root Mean Square - RMS) e o nível de sobrecarga. Entretanto, após um determinado nível de sobrecarga, um aumento exponencial do sinal EMG pode ser notado.

Esse ponto de quebra da linearidade é chamado de Limiar de Fadiga Eletromiográfico (LFEMG) (HUG et al., 2006a; HUG et al., 2003). A proposta de determinação do LFEMG surgiu com o intuito de estimar o Limiar Ventilatório (LV) de maneira mais simples e com custos financeiros reduzidos, já que o analisador de gases inspirados possui um custo muito mais elevado quando comparado a um eletromiógrafo. Vale ressaltar ainda que uma grande vantagem de LFEMG sobre LV seria a possibilidade de identificar o comportamento localizado de diversos músculos, já que o LV oferece uma informação generalizada sobre a capacidade aeróbia do indivíduo.

O LFEMG tem sido associado ao ponto de transição do metabolismo aeróbio-anaeróbio (HUG et al., 2003, JURIMAE et al., 2007) e tem se mostrado relacionado a outros índices de capacidade aeróbia e desempenho (MAESTU et al., 2006). Vários estudos têm utilizado protocolos em cicloergômetro para analisar as respostas da EMG obtida em músculos dos membros inferiores (HUG et al., 2006a; HUG et al., 2003; VERCRUYSSEN, MISSENARD \& BRISSWALTER, 2009), no entanto, pouco se sabe quanto a ativação muscular durante protocolo incremental em cicloergometro de membro superior (HINTZY, TORDI \& PERREY, 2002; VERGÈS et al., 2009). 
Smith et al. (2008) relatam que durante o exercício no ergômetro de braço os principais músculos agonistas são os do braço e do ombro. Os autores ainda afirmam que o Bíceps Braquial (BB) e o Tríceps Braquial (TB) atuam em fases antagônicas do ciclo, além disso, a atividade dos músculos do ombro ocorre em momentos diferentes da revolução da manivela quando comparada à dos músculos do braço citados anteriormente. No entanto, num protocolo de testes incremental o padrão de recrutamento das unidades motoras pode ser modificado e comprometer essa coordenação refinada. Dessa forma, o objetivo desse estudo foi verificar a ativação de quatro músculos do membro superior (Bíceps Braquial, Tríceps Braquial, Deltóide Anterior e Deltóide Posterior) e determinar o LFEMG durante um teste incremental em cicloergômetro de braço.

\section{Métodos}

\section{Sujeitos}

Quatro indivíduos saudáveis do sexo masculino (idade: 25,25 $\pm 4,7$; altura: $173 \pm 4 \mathrm{~cm}$; massa corporal: $71,5 \pm 6,3 \mathrm{~kg}$ ). Todos os sujeitos eram fisicamente ativos (um atleta de natação e três praticantes de musculação e/ou corrida), com carga de treino semanal de $5 \mathrm{~h}$ a 12h. Nenhum deles havia tido experiência prévia com ergômetro de braço. 0 experimento foi conduzido de acordo com as Diretrizes e Normas de Pesquisa em Seres Humanos, e todos os voluntários foram informados dos objetivos da pesquisa e concordaram em participar assinando um termo de consentimento.

\section{Protocolo}

Todos os sujeitos realizaram um teste incremental no cicloergômetro de braço com freio elétrico-magnético (Handcycle 300-E2, Endorphin Corporation, EUA), com incrementos de carga de 3-4 W a cada 4 min, começando com $19 \mathrm{~W}$ e terminando com $48 \mathrm{~W}$ (potência máxima do ergômetro). Não houve aquecimento prévio, pois a carga inicial foi baixa. 0 término do teste ocorreu quando foi alcançado o máximo de potência do ergômetro.

Os testes foram realizados no mesmo dia e período. Os sujeitos fizeram os testes sem camisa, para facilitar a visualização do posicionamento dos eletrodos no decorrer do teste. A cadência estabelecida foi de $80 \pm 5 \mathrm{rpm}$. 0 monitor da cadência foi posicionado de frente para o sujeito durante cada teste e um pesquisador monitorou a manutenção da cadência dentro da faixa estabelecida ao longo do teste. Antes do começo de cada experimento, o posicionamento da cadeira e do encosto peitoral foi ajustado e padronizado entre os participantes, no qual no ponto mais distal do ciclo (pé de vela paralelo ao solo e manopla no ponto mais distante do tronco do indivíduo) o braço deveria encontrar-se levemente flexionado e a mão levemente abaixo da linha do ombro.

\section{Coleta e Processamento da EMG}

Durante cada teste, a atividade eletromiográfica de quatro músculos do membro superior direito foi coletada: Bíceps Braquial (BB), Tríceps Braquial (TB - cabeça longa), Deltóide Anterior (DA), Deltóide Posterior (DP). Os dados sobre a ativação muscular foram obtidos por meio de um eletromiógrafo Myosystem 2000 (Noraxon, EUA). 0 mesmo apresenta razão de rejeição em modo comum de $114 \mathrm{~dB}$, impedância de entrada entre $20 \mathrm{M} \Omega$ e $1 \mathrm{G} \Omega$ e largura de faixa entre 16 e $500 \mathrm{~Hz}$.

Os sinais eletromiográficos foram coletados a uma freqüência de $1.000 \mathrm{~Hz}$ através do programa de aquisição de dados MyoResearch versão 2.10 (Noraxon, EUA). Os sinais foram armazenados em um computador para posterior visualização e análise. Para cada músculo, foi fixado à pele um par de eletrodos de superfície com sensor Ag/AgCl (ECG Electrode, Kobme, Coréia do Sul) com distância fixa de $2 \mathrm{~cm}$ entre os centros dos eletrodos. Os eletrodos foram posicionados segundo as regras estabelecidas pelo SENIAM, respeitando a direção das fibras musculares de cada músculo.

Previamente à colocação dos eletrodos, a pele foi limpa com álcool, mas não foi necessário raspagem, pois não há muitos pelos nas áreas dos músculos escolhidos para o experimento.

Os eletrodos foram fixados com ajuda de esparadrapo (Micropore, 3M, Brasil) para evitar alguma mudança no posicionamento dos eletrodos durante a atividade. A RMS foi 
calculada de acordo com a média do sinal EMG a cada 5 segundos. A Figura 1 apresenta um exemplo de sinal pré e pós tratamento.

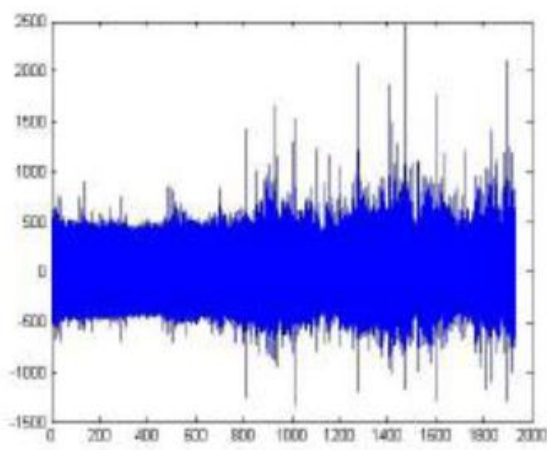

(a)

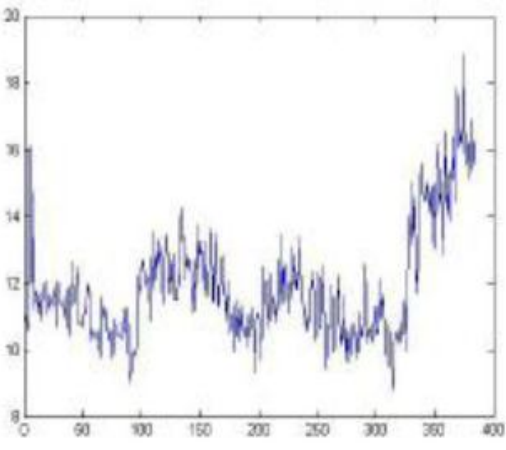

(b)

Figura 1- Exemplo dos sinais EMG pré (item A) e pós tratamento (item B) coletados de um dos voluntários durante o teste no ciclo ergômetro.

\section{Determinação do LFEMG}

O Limiar de Fadiga Eletromiográfico (LFEMG) foi detectado através da análise visual dos pesquisadores envolvidos no presente estudo. Hug et al. (2006b) demonstrou que a determinação do LFEMG pelo método de detecção visual é fidedigno e condiz com a determinação desse fenômeno por meio de algoritmos de regressão.

\section{Coleta da Freqüência Cardíaca}

Durante os testes, todos os voluntários tiveram a freqüência cardíaca monitorada por meio de um monitor cardíaco (F5, Polar, EUA). Antes de cada teste a pele dos sujeitos foi limpa e esfoliada no local em que seria acoplado o cinto transmissor. 0 gel condutor foi posto sobre a superfície do cinto que entraria em contato com a pele do indivíduo, para melhorar a coleta dos dados. A FC foi coletada a cada 4 minutos, imediatamente antes de cada aumento de potência durante o protocolo de carga incremental escolhido para a pesquisa.

\section{Resultados}

Os resultados apresentaram uma alta variabilidade inter-sujeitos e inter-músculos. Porém, houveram tendências no comportamento do sinal EMG nas duas porções do deltóide (DA e DP), apresentando diminuição de ativação ao longo do teste. A figura 2 e 3 apresentam valores individuais de EMG para os músculos DA e DP, respectivamente, durante o teste incremental. 


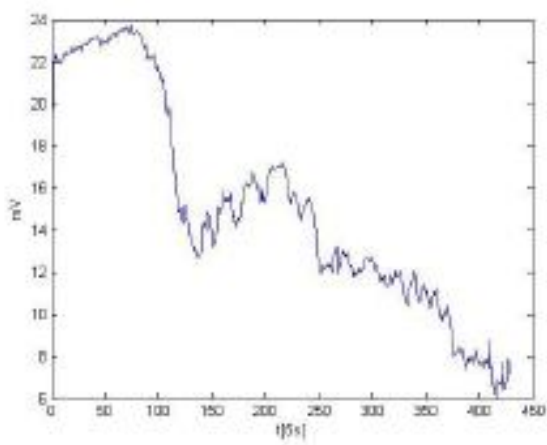

(a)

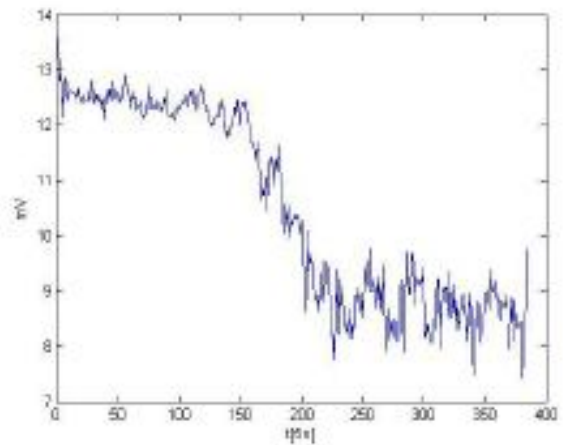

(c)

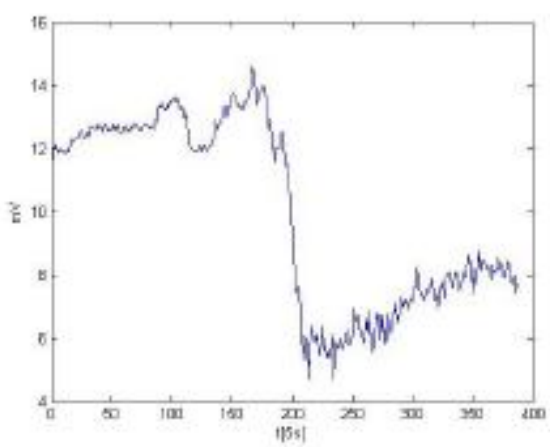

(b)

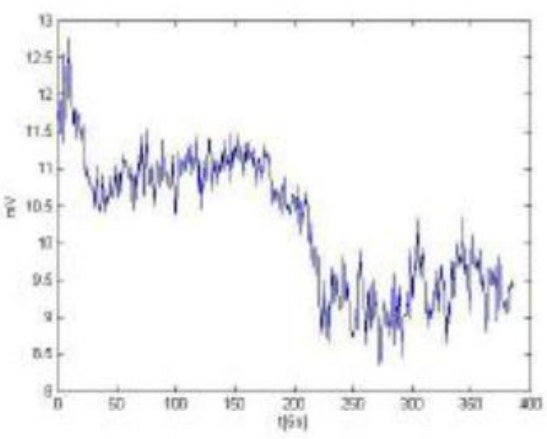

(d)

Figura 2 - Resposta eletromiográfica individual do músculo Deltóide Anterior (DA) durante o teste incremental. a - sujeito I; b - sujeito II; c - sujeito III; d - sujeito IV.

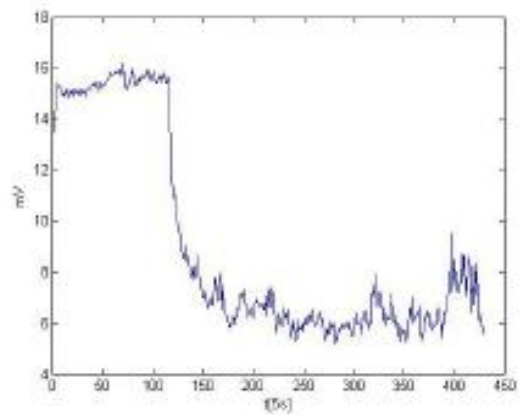

(a)

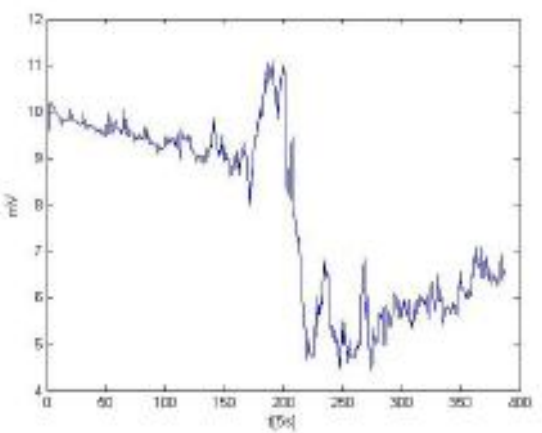

(b) 


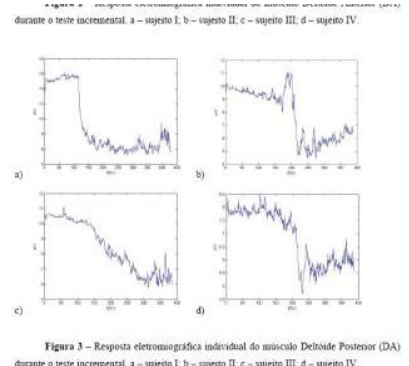

(c)

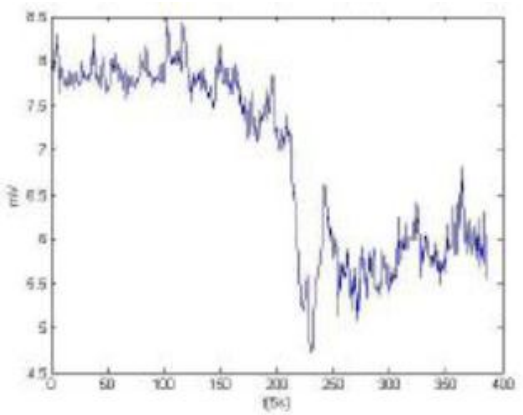

(d)

Figura 3 - Resposta el etromiográfica individual do músculo Deltóide Posterior (DA) durante o teste incremental. a - sujeito I; b - sujeito II; c - sujeito III; d - sujeito IV.

Para TB, o sinal EMG apresentou menor variância, no entanto, também foi possível observar quedas de ativação. A figura 4 apresenta os valores individuais de EMG para os músculos TB durante o teste incremental.

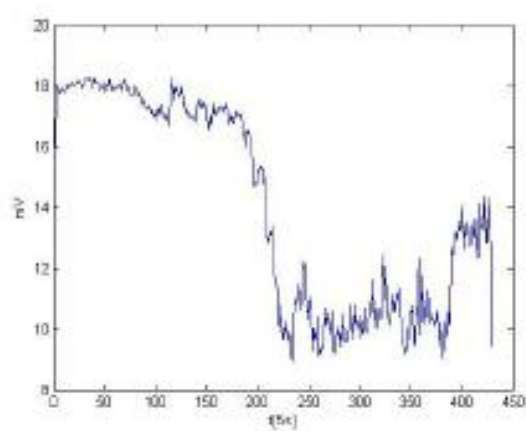

(a)

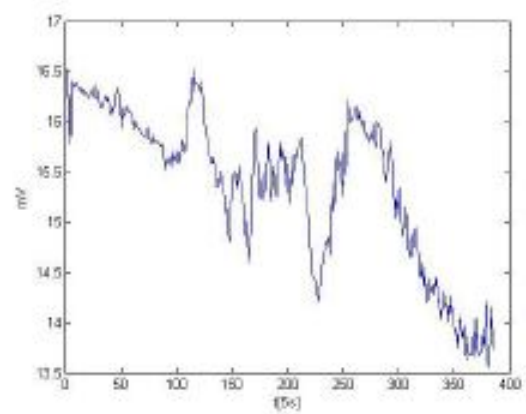

(c)

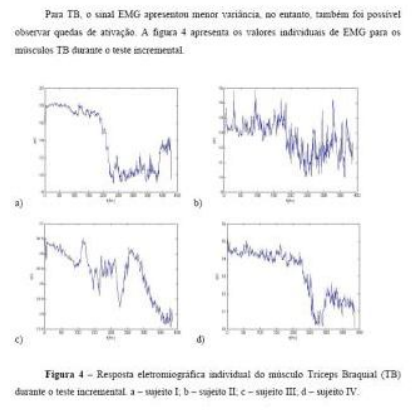

(b)

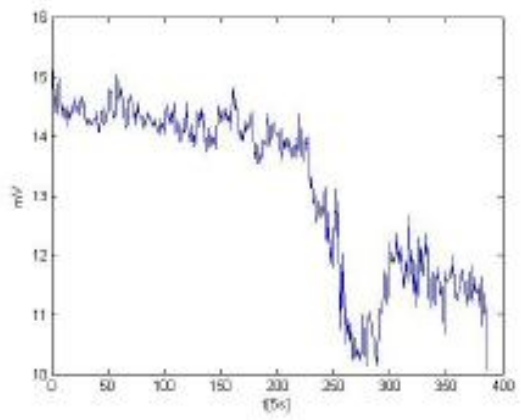

(d)

Figura 4 - Resposta el etromiográfica individual do músculo Tríceps Braquial (TB) durante o teste incremental. a - sujeito I; b - sujeito II; c - sujeito III; d - sujeito IV.

Já para BB, a ativação aumentou em todos os sujeitos, ainda que esse aumento tenha sido menos acentuado em alguns casos. A figura 5 demonstra os valores individuais de EMG para os músculos BB durante o teste incremental. 


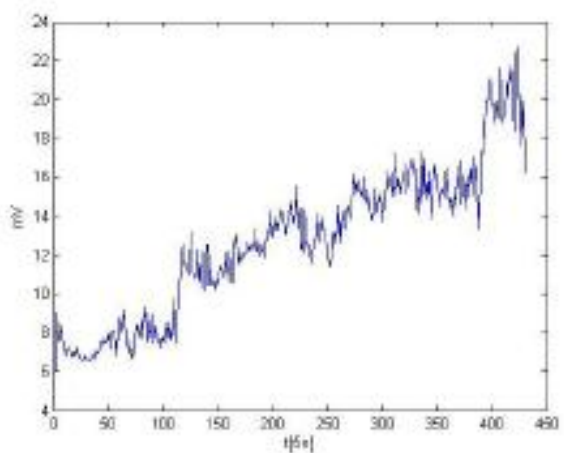

(a)

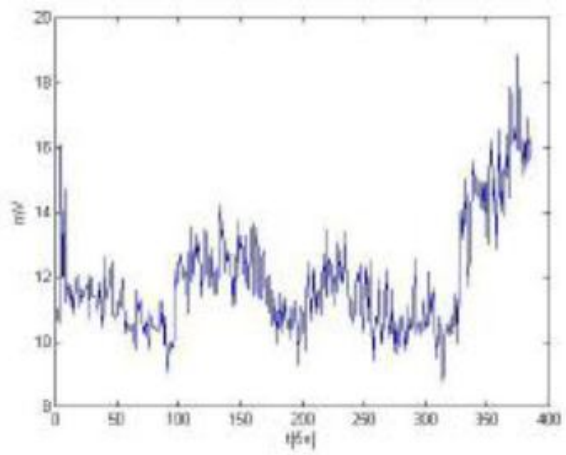

(c)

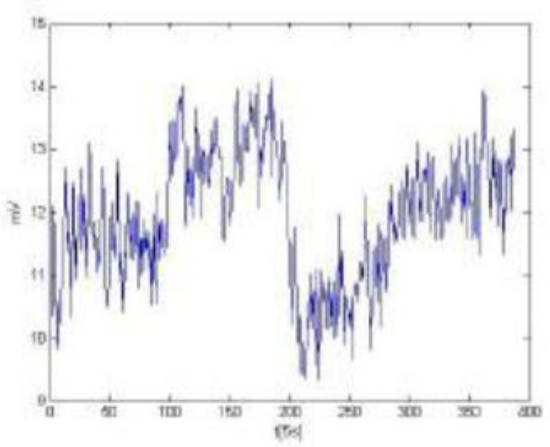

(b)

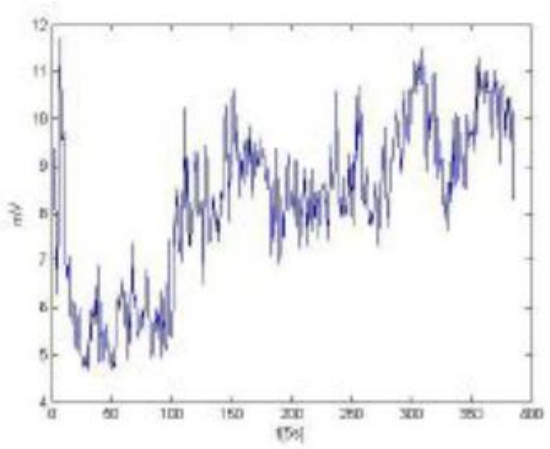

(d)

Figura 5 - Resposta eletromiográfica individual do músculo Bíceps Braquial (TB) durante o teste incremental. a - sujeito I; b - sujeito II; c - sujeito III; d - sujeito IV.

O LFEMG não foi possível de ser determinado na maioria dos casos. De 16 possíveis detecções desse fenômeno (4 músculos em 4 sujeitos), o LFEMG foi encontrado em apenas um músculo (BB) de um dos voluntários, correspondendo a apenas 6,25\% do total. 0 LFEMG foi detectado com 27 minutos de teste, a uma potência de $44 \mathrm{~W}$ (aproximadamente $90 \%$ da potência máxima do ergômetro). Na figura 6 é apresentado o único LFEMG encontrado entre os sujeitos e músculos analisados durante o teste de carga incremental.

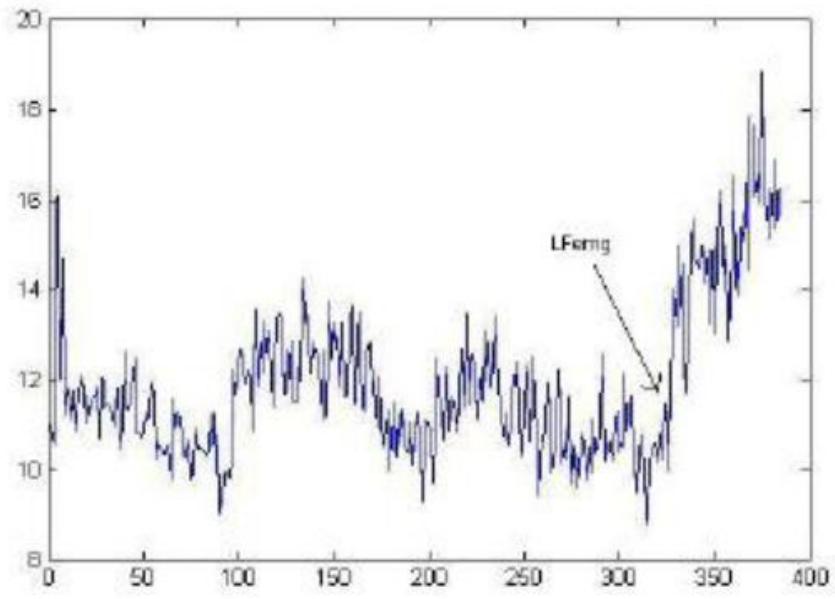

Figura 6 - Determinação do LFemg no músculo Bíceps Braquial (BB) de um dos voluntários da pesquisa 
Durante o teste incremental foi também monitorada a freqüência cardíaca (FC). Podemos observar que a FC aumenta linearmente em função do tempo e do aumento da potência (intensidade). Com término de 32 minutos de teste para todos os sujeitos, a freqüência não passou dos $60 \%$ da freqüência cardíaca máxima estimada dos indivíduos. A figura 7 apresenta a resposta da FC em boxplot dos voluntários da pesquisa durante o teste incremental.

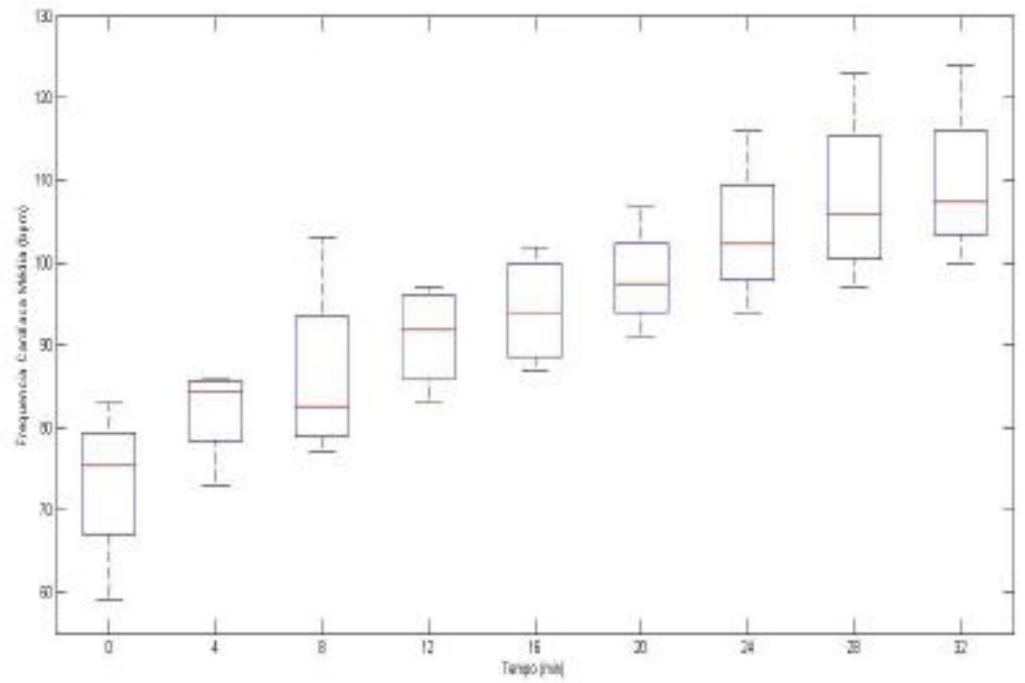

Figura 7 - Boxplot com os valores da freqüência cardíaca (FC) dos voluntários coletados antes do início e a cada 4 minutos do teste.

\section{Discussão}

0 presente estudo aparece como um dos pioneiros referentes à análise da ativação muscular e determinação do LFEMG em músculos dos membros superiores durante um protocolo de carga incremental em cicloergômetro de braço. Os músculos analisados apresentaram alta variação quanto as suas ativações com o incremento da carga durante o teste, e, apontam para um comportamento diferenciado do padrão de recrutamento com o aumento da carga imposta, já que a variação entre os sujeitos foi relativamente menor. Em relação a determinação do LFEMG, esse apresentou uma incidência muito baixa, sendo encontrado em apenas um caso no músculo BB.

A queda da ativação dos deltóides sugere que esses músculos podem ter atuado majoritariamente na estabilização do ombro (POLITTI et al., 2006), ainda que o serrátil anterior tenha sido apontado por Kiyomoto et al. (2007) como o principal estabilizador dessa articulação. É proposto também que o DA pode agir como um dos músculos motores primários da flexão horizontal de ombro (AABERG, 2002), e sua maior atividade no começo em relação ao final do teste pode ter ocorrido devido a inércia inicial não ser suficiente para finalizar o ciclo e recomeçar a fase de propulsão do movimento.

O DP, por sua vez, é um dos principais atuantes na extensão do ombro (AABERG, 2002). É possível que a queda de seu recrutamento seja conseqüência do aumento da atuação do BB. No cicloergômetro, o momento de extensão do ombro é praticamente concomitante à flexão de cotovelo, portanto, se o torque gerado pelo esforço do BB (flexor do cotovelo e maior propulsor do movimento) for grande, pode atenuar a necessidade de atuação do DP. Por outro lado, Kawano et al. (2007) demonstraram que durante um exercício de abdução do ombro no plano frontal até a exaustão ocorre um decréscimo na freqüência mediana do sinal EMG nos músculos DA e DP, caracterizando a fadiga periférica. Se considerarmos que a abdução frontal do ombro é um movimento que compõe a revolução do cicloergômetro de braço, podemos sugerir que a queda de ativação de DA e DP pode ter sido conseqüência da fadiga periférica. 
No entanto, a freqüência mediana do sinal eletromiográfico não foi aferida no presente estudo, impossibilitando maiores conclusões.

Em relação ao comportamento de TB, nossos dados nos levam a crer que a diminuição da ativação desse músculo está diretamente relacionada ao maior recrutamento contralateral do BB durante cargas mais elevadas no ciclo ergômetro de braço. Segundo Smith et al. (2008), o pico de torque no cicloergômetro de braço ocorre na fase de "puxada" do ciclo. Se observarmos bem, na fase de puxada a ação principal é a flexão de cotovelo e um dos músculos responsáveis por esse movimento é o BB. Provavelmente o BB é o principal propulsor do movimento realizado no teste e o comportamento da ativação dele relatado durante cargas mais elevadas neste estudo apenas reforça essa hipótese. Smith et al. (2008) ainda relatam que o TB age no ciclo em um momento antagônico ao BB. Se levarmos em conta a sincronia das manivelas, podemos observar que a atuação do TB é aliviada com o pico de torque gerado pelo BB contralateral.

Quanto ao LFEMG, foi possível determiná-lo em apenas uma ocasião, no músculo BB. Assim como Hug et al. (2006a) analisando a ocorrência do LFEMG em membros inferiores durante o ciclismo, ficou evidente que esse fenômeno é mais fácil de ser determinado em músculos importantes na propulsão da ação realizada. Esse achado corrobora a hipótese que o BB é o principal propulsor das revoluções no cicloergômetro de braço. A detecção do LFEMG pode ter sido influenciada pela diferença do nível de treinamento dos indivíduos, pois o individuo que apresentou LFEMG também praticava natação, e, diferentemente dos outros voluntários praticantes de musculação, o nadador está mais acostumado a movimentos cíclicos com os membros superiores.

A técnica utilizada na execução do movimento também pode ter influenciado a determinação de LFEMG, pois o giro exigido no ergômetro de braço nunca havia sido praticado por nenhum dos sujeitos e nem mesmo houve contato prévio para familiarização com o aparelho e com o movimento. A curta duração do teste e o baixo pico de potência do ergômetro também podem ter dificultado o aparecimento do LFEMG, que talvez pudesse ser encontrado com o aumento do tempo gasto em cada estágio e/ou da intensidade do esforço.

Já a variação da FC ao longo do teste foi pequena, fato que pode ser creditado ao baixo pico de potência do ergômetro utilizado neste estudo. Também houve pequena variação da FC entre os sujeitos, fato que pode ser creditado à semelhança em suas idades. Com a intensidade reduzida do esforço, a FC não variou muito e mesmo no último estágio do teste os voluntários não chegaram perto do máximo esforço subjetivo. Possivelmente com uma freqüência de ciclos maior e um ergômetro com pico de potência maior, o LFEMG poderia ser determinado mais corriqueiramente.

\section{Conclusão}

Com base em nossos experimentos, observamos que o comportamento da atividade EMG variou de acordo com a função do músculo na mecânica do movimento, por conta disso, podemos sugerir que o BB é o principal músculo propulsor na execução dos movimentos no cicloergômetro de braço. Por conta dessa maior ativação, a tendência é que os outros músculos (TB, DA e DP) sejam menos recrutados e haja maior facilidade de detecção do LFEMG no BB, assim como evidenciado em nossos achados. Pesquisas mais aprofundadas serão necessárias para esclarecer se existem outros músculos com atividade semelhante à do BB, que possam ser considerados como auxiliares na propulsão. A partir disso, a utilização do cicloergômetro poderá ser mais direcionada, tanto para avaliação do condicionamento físico quanto para reabilitação. 


\section{Referências}

AABERG, E. Conceitos e técnicas para o treinamento resistido. São Paulo-SP: Manole, 2002.

BARBOSA, F. S. \& GONÇALVES, M. “Comparação entre protocolos de exaustão e de 30 segundos utilizados na avaliação da fadiga eletromiográfica dos músculos eretores da espinha”, Rev. Brasileira de Fisioterapia, 9(1): 77-83. 2005.

GABRIEL, D. A.; BASFORD, J. R.; AN, K. "Training-related changes in the maximal rate of torque development and EMG activity”, Journal of Electromyography and Kinesiology, 11: 123-29. 2001.

GONÇALVES, M. "Eletromiografia e a identificação da fadiga muscular", São Paulo-SP: Rev. Bras. Educ. Fís. Esp., 20(Suplemento \# 5): 91-93. 2006.

HINTZY, F.; TORDI, N.; PERREY, S. "Muscular Efficiency During Arm Cranking and Wheelchair Exercise: a comparison", International Journal of Sports Medicine, 23(6): 408-14. 2002.

HUG, F.; FAUCHER, M.; KIPSON, N.; JAMMES, Y. "EMG signs of neuromuscular fatigue related to the ventilatory threshold during cycling exercise", Clinical Physiology and Functional Imaging, 23(4): 208-14. 2003.

HUG, F.; LAPLAUD, D.; LUCIA, A.; GRELOT, L. “EMG Threshold Determination in Eight Lower Limb Muscles During Cycling Exercise: a pilot study", International Journal of Sports Medicine, 27(6): 45662. $2006 a$.

HUG, F; LAPLAUD, D; LUCIA, A; GRELOT, L. “A comparison of visual and mathematical detection of the electromyographic threshold during incremental pedaling exercise: a pilot study", Journal of Strength and Conditioning Research, 20(4): 704-08. 2006b.

JÜRIMÄE, J.; VON DUVILlARD, S.P.; MÄESTU, J.; CICCHELlA, A.; PURGE, P.; RUOSI, S.; JÜRIMÄE, T.; HAMRA, J. "Aerobic-anaerobic transition intensity measured via EMG signals in athletes with different physical activity patterns”, European Journal of Applied Physiology, 101: 341-46. 2007.

KAWANO, M. M.; PESSAN, M. A.; RODRIGUES, D.; STABILE, G. R.; CARDOSO, J. R.. “Comportamento da fadiga das três porções do deltóide durante a abdução no plano escapular e frontal”, In: XII Congresso Brasileiro de Biomecânica, 2007, São Pedro. Anais do XII Congresso Brasileiro de Biomecânica. pp. 1256-61.

KIYOMOTO, H.; GUERREIRO, P; ARAUJO, R.; MOCHIZUKI, L. “Análise Biomecânica dos Músculos Estabilizadores do ombro", In: XII Congresso Brasileiro de Biomecânica, 2007, São Pedro. Apresentação de Trabalho/Seminário.

MAËSTU, J.; CICCHELLA, A.; PURGE, P.; RUOSI, S.; JURIMAE, J.; JURIMAE, T. “Electromyographic and neuromuscular fatigue thresholds as concepts of fatigue", Journal of Strength and Conditioning Research, 20: 824-28. 2006.

OLIVEIRA, A.S.C.; GONÇALVES, M.; CARDOZO, A.C.; BARBOSA, F. S. S “Electromyographic fatigue threshold of the biceps brachii muscle during dynamic contraction", Electromyogr. clin. Neurophysiol, 45: 167-75. 2005.

POLITTI, F.; PALOMARI, E.T.; RICARDO FURTADO, R.; CÉSAR FERREIRA AMORIM, C. F. “Análise eletromiográfica e da força do músculo deltóide em indivíduos com síndrome do impacto do ombro", Fisioterapia e Pesquisa, 13(3): 24-31. 2006.

SILVA, S. R.; GONÇALVES, M. “Comparação de Protocolos para Verificação da Fadiga Muscular pela Eletromiografia de Superfície”, Motriz, 9(1): 51-58, 2003.

SMITH, P.; CHAPMAN, M.; HAZLEHURST, K.; GOSS-SAMPSON, M. “The influence of crank configuration on muscle activity and torque production during arm crank ergometry", Journal of Electromyography and Kinesiology, 18(4): 598-605. 2008.

VERCRUYSSEN, F; MISSENARD, O; BRISSWALTER, J. "Relationship between oxygen uptake slow component and surface EMG during heavy exercise in humans: Influence of pedal rate”, Journal of Electromyography and Kinesiology, 19(4): 676-84. 2009.

VERGÈS, S.; FLORE, P.; NANTERMOZ, G.; LAFAIX, P. A.; WUYAM, B. "Respiratory Muscle Training in Athletes with Spinal Cord Injury", International Journal of Sports Medicine, 30(7): 526-32. 2009. 